\title{
Formoterol as relief medication in asthma: the jury is still out
}

\author{
C. Jenkins
}

Despite concerns that use of long-acting $\beta$-agonists (LABA) could be associated with risk of worsening asthma, to date these drugs have proven safe and highly effective $[1,2]$. Early studies demonstrated no increase in asthma exacerbations when added to inhaled corticosteroids (ICS) as long-term maintenance medication and subsequent studies have shown significantly reduced risks of exacerbations and improved, more rapid establishment of asthma control compared to adding more ICS [3-5]. Formoterol is a potent LABA with a rapid onset of bronchodilatation [6, 7] and a relatively fast clinical response when added as regular medication for patients who are symptomatic on ICS alone $[5,8,9]$. The question therefore arises, could formoterol with its rapid onset of action, also be used safely and effectively as reliever medication for asthma [10]? Are there risks of tolerance associated with regular use of formoterol that could reduce its benefit when used as a reliever for acute symptoms [11]? Are there other risks, associated with its adrenergic and metabolic effects, that could be increased by its use when added to regular formoterol, resulting in worse outcomes [12]?

Some of these questions have already been addressed in two 12 week randomised controlled trials. TATTERSFIELD et al. [13] showed in a double-blind study in 362 symptomatic patients taking ICS (mean daily dose $870 \mu \mathrm{g}$ ) that formoterol $4.5 \mu \mathrm{g}$ as reliever medication was associated with fewer asthma exacerbations and a longer time to first exacerbation compared to terbutaline $500 \mu \mathrm{g}$. Serum potassium and electrocardiogram variables were monitored at clinic visits and no differences between treatments were shown. The relative risk ratio for an exacerbation requiring oral corticosteroids was $0.55 \quad(95 \%$ confidence interval $0.34-0.89)$. The average daily dose of formoterol was four puffs of $4.5 \mu \mathrm{g}$, and this did not change over the period of the study. In a double-blind randomised controlled trial in 357 symptomatic patients with moderate asthma taking ICS and regular formoterol $9 \mu \mathrm{g}$ b.i.d., IND et al. [14] compared the addition of formoterol $4.5 \mu \mathrm{g}$ or terbutaline $500 \mu \mathrm{g}$ as needed over 12 weeks. Adverse events, electrocardiograms and serum potassium were monitored. Both treatments were equally safe and there was no significant difference in severe exacerbation rates between treatment groups. On average patients used 2 puffs of formoterol $4.5 \mu \mathrm{g}$ as reliever medication daily, and $20 \%$ patients had an exacerbation requiring oral steroids, with no significant differences between treatments. These authors concluded that formoterol could replace short-acting $\beta$-agonists (SABA) as reliever medication, and that the concept merited testing in a "real-world" setting. More recently, some concern has been raised from preliminary reports about the risk of exacerbation with regular LABA in particular subgroups of patients [15] or

Correspondence: C. Jenkins, Head, Asthma Group, Woolcock Institute of Medical Research and Co-operative Research Centre for Asthma, PO Box M77, Missenden Rd, Camperdown NSW 2050, Australia. Fax: 61 299290143. E-mail: crj@med.usyd.edu.au at high doses [16], although more information from these studies is needed.

The safety of formoterol as a reliever has now been further addressed by one of the largest studies ever undertaken in asthma, published in the European Respiratory Journal this month [17]. The study of formoterol as relief medication in asthma, was a "real-world", open label, safety and effectiveness trial. The strength of the study is its size and the wide range of patients who were eligible, from those with very mild asthma on no regular ICS through to those with more severe disease, assessed according to ICS use at entry and the Global Initiative for Asthma (GINA) guidelines. Just over 18,000 patients were randomised to receive either formoterol $4.5 \mathrm{mcg}$ or salbutamol $200 \mathrm{mcg}$ as relief medication to be used as needed for 6 months. At entry $76 \%$ of the patients were taking ICS and $31 \%$ were taking LABA, representing a broad spectrum of asthma severity.

There were more withdrawals in the formoterol group due to asthma and nonasthma-related adverse events (AE), although this should be interpreted in the light of the open-label study design. Nonasthma-related AEs (tremor, headache and tachycardia) increased with patients' age. There were no differences in serious AEs, but there were significantly fewer asthma-related AEs in the formoterol group. In the formoterol arm fewer patients experienced an asthma exacerbation and it took longer to first exacerbation. The study therefore provides new information suggesting that formoterol is a safe medication for use as a reliever in a wide range of asthma patients. It adds to the findings of TATTERSFIELD et al. [13] who showed in a double-blind 3 month study that formoterol as reliever medication was associated with fewer asthma exacerbations compared to terbutaline.

Although the size of the study particularly offers some reassurance about safety, two significant weaknesses in design mean that caution must be exercised in interpreting the results, especially in regard to the effects on exacerbations. First, exacerbations were assessed subjectively with no measure of daily peak flow or clinic spirometry. Exacerbations were defined by several criteria including investigator decision to increase maintenance treatment or prescribe oral corticosteroids. As acknowledged by the authors, the investigators knew which study drug the patient was taking, introducing significant potential for bias. One of the primary outcomes, exacerbation rate, depended on the investigator's decision regarding the need for extra maintenance medication or oral corticosteroids. Potentially, symptomatic patients in the formoterol arm might have been less likely to be offered increased medication, therefore not qualifying as having an exacerbation. Bias could have operated in the other direction if the investigators believed there were risks associated with formoterol. Secondly, patients were able to take up to 12 puffs of extra formoterol or salbutamol a day before contacting the investigator. Based on the dose response curves for these two $\beta$-agonists, formoterol being a full agonist and having a prolonged effect, this is not an equivalent dose. 
Investigator contact and recording of an exacerbation would be more likely to occur on this basis in the salbutamol arm. While the greater benefit of up to 12 puffs of formoterol would be a true positive effect of formoterol over salbutamol, this aspect of study design would favour the formoterol arm in the efficacy assessment.

As the study was not double blind nor placebo controlled, it provides Level III evidence that formoterol as relief medication is safe and may be associated with fewer exacerbations and daily asthma symptoms. Evidence from the previous studies of TATTERSFIELD et al. [13] and IND et al. [14], provide level II evidence for the safety of formoterol as reliever medication in patients on regular ICS, with or without regular LABA.

Before declaring on the basis of these studies that formoterol is safe as a reliever medication, the implications of its use in this way should be considered. Exacerbations are at the far end of the continuum of reliever use for symptoms. If generally taken "as-needed" for relief, patients would probably increase the dose and frequency of use during exacerbations.

In view of the high likelihood of this, formoterol should be shown to be at least as safe and effective during exacerbations as SABA, the current treatment for acute symptoms during exacerbations $[10,18]$. The possibility that formoterol could be used in exacerbations both to relieve increasing asthma symptoms and to achieve more rapid control of deteriorating asthma is suggested by the relatively fast response when LABA are added as regular medication for patients who are symptomatic on ICS alone [5-8]. In vitro studies and some clinical studies also suggest a synergistic effect for LABA with ICS that may be advantageous during asthma exacerbations and periods of poor asthma control [19-21]. However, if formoterol were used as relief medication during an exacerbation, failure to respond or persistent symptoms could result in excessive dosing and increased risk of poorer outcomes. This may especially be true for patients with severe or poorlycontrolled disease. In the published randomised trials to date there is no evidence of an excess of severe exacerbations, hospital admissions or life-threatening asthma for LABA compared to SABA as reliever. As a significant proportion of patients in the study by PAUWELs et al. [17] had exacerbations, some must have taken the maximum permitted dose of formoterol (12 puffs a day). It would be helpful to know if discontinuations due to adverse events such as tremor and tachycardia were related to the number of puffs of formoterol taken.

There are now several studies of formoterol as reliever medication in patients with asthma representing all but those with very severe disease. Does more need to be done in order to establish formoterol as a possible alternative to shortacting $\beta$-agonists for reliever medication? Probably not with respect to safety, if recommendations for its use are carefully prescribed and confined to the circumstances in which it has been trialled. It should first be asked however, whether this is necessary and what gains are to be made? There needs to be clear benefit, since changes in the management of potentially unstable asthma must be approached with great care. If the gains are in adherence to treatment regimens by reducing the number of inhalers needed, the benefits and cost of this approach need to be demonstrated. If the gains are in improving asthma symptom control and reducing exacerbations, then a longer, double-blind placebo controlled study needs to be done. Optimally a trial should be undertaken to assess the safety and effectiveness of formoterol in the outpatient management of exacerbations. Until then, formoterol as relief medication should be regarded as safe but its effectiveness over as-needed short-acting $\beta$-agonists is not yet supported by level I evidence.

\section{References}

1. Kips JC, Pauwels RA. Long acting inhaled beta-agonist therapy in asthma. Am J Respir Crit Care Med 2001; 164: 923-932.

2. Shrewsbury S, Pyke S, Britton M. Meta-analysis of increased doses of inhaled steroid or addition of salmeterol in symptomatic asthma (MIASMA). BMJ 2000; 320: 1368-1373.

3. Greening AP, Ind PW, Northfield M, Shaw G. Added salmeterol versus higher-dose corticosteroids in asthma patients with symptoms on existing inhaled corticosteroids. Lancet 1994; 344: 219-224.

4. Woolcock A, Lundback B, Ringdal N, Jacques LA. Comparison of addition of salmeterol to inhaled steroids with doubling of the dose of inhaled steroids. Am J Respir Crit Care Med 1996; 153: 1481-1488.

5. Pauwels RA, Lofdahl CG, Postma DS, et al. Effect of inhaled formoterol and budesonide on exacerbations of asthma. N Engl J Med 1997; 337: 1405-1411.

6. Van Noord JA, Smeets JJ, Raaijmakers JA, Bommer AM, Maesen FPV. Salmeterol versus formoterol in patients with moderately severe asthma; onset and duration of action. Eur Respir J 1996; 9: 1684-1688.

7. Palmqvist M, Persson G, Lazer L, Rosenborg J, Larsson P, Lotvall J. Inhaled dry powder formoterol and salmeterol in asthmatic patients: onset of action, duration of effect and potency. Eur Respir J 1997; 10: 2484-2489.

8. Zetterstrom $\mathrm{O}$, Buhl $\mathrm{R}$, Mellum $\mathrm{H}$, et al. Improved asthma control with budesonide/formoterol in a single inhaler compared with budesonide alone. Eur Respir J 2001; 18: 262-268.

9. Schreurs AJM, Sinninghe Damste HEJ, de Graaf CS, Greefhorst APM. A dose response study with formoterol Turbuhaler as maintenance therapy in asthmatic patients. Eur Respir J 1996; 9: 1678-1683.

10. Malolelepsky J, Boszormenyi Nagy G, Selroos O, Larsson P, Brander R. Safety of formoterol turbuhaler at cumulative dose of $90 \mathrm{mg}$ in patients with acute bronchial obstruction. Eur Respir J 2001; 18: 928-934.

11. Lipworth BJ, Aziz I. Bronchodilator response to albuterol after regular formoterol and effects of acute corticosteroid administration. Chest 2000; 117: 156-162.

12. Nelson HS. $\beta$-adrenergic bronchodilators. New Engl J Med 1995; 333: 499-506.

13. Tattersfield AE, Lofdahl C-G, Postma DS, et al. Comparison of formoterol and terbutaline for as-needed treatment of asthma: a randomised controlled trial. Lancet 2001; 357: 257-261.

14. Ind PW, Villasante C, Shiner RJ, et al. Safety of formoterol by Turbuhaler as reliever medication compared to terbutaline in moderate asthma. Eur Respir J 2002; 20: 859-866.

15. The FDA Safety Information and Adverse Reporting Program. 2003 Safety Alert - Serevent (salmeterol xinafoate). www.fda.gov/medwatch/SAFETY/2003/serevent.htm. Date updated: January 23 2003; Date accessed: February 32003.

16. Mann M, Chowdhury B, Sullivan E, Nicklas R, Anthracite $\mathrm{R}$, Meyer RJ. Serious asthma exacerbations in asthmatics treated with high dose formoterol. Chest 2003; 124: 70-74.

17. Pauwels RA, Sears MR, Campbell M, et al. Formoterol as relief medication in asthma: a worldwide safety and effectiveness trial. Eur Respir $J$ 2003; 22: 787-794.

18. Kelly HW, Murphy S. Beta-adrenergic agonists for acute severe asthma. Ann Pharmocother 1992; 2681-91.

19. Roth M, Johnson PRA, Rudiger JJ, et al. Interaction between glucocorticoids and $\beta_{2}$ agonists on bronchial airway smooth muscle cells through synchronised cellular signalling. Lancet 2002; 360: 1293-1299.

20. Pang L, Knox AJ. Synergistic inhibition by $\beta$-agonists and corticosteroids on tumour necrosis factor- $\alpha$-induced interleukin-8 release from cultured human airway smooth muscle cells. Am J Respir Cell Mol Biol 2000; 23: 79-85.

21. Barnes PJ. Scientific rationale for inhaled combination therapy with long acting $\beta_{2}$-agonists and corticosteroids. Eur Respir J 2002; 19: 182-191. 